\title{
The internal thoracic (mammary) nerve
}

\author{
A. A. PEAR SON and R. W. SA U TER \\ Department of Anatomy, University of Oregon Medical School, Portland, Oregon, USA 97201
}

The internal thoracic (mammary) nerve is formed by contributions from the subclavian plexus and the phrenic nerve. It descends along the internal thoracic artery to the level of the seventh costal cartilage where it is lost. Some of its branches communicate with the intercostal nerves and are thought to be distributed with these nerves. Others follow perforating and anterior mediastinal arteries.

This study is based on human embryos and fetuses which were sectioned serially and prepared with neurological stains. These include the protargol method of Bodian (1936), the silver method of Pearson and Whitlock (1949), and other staining procedures developed in this laboratory by the authors. Twenty-five human specimens which ranged in size from 12 to 111 mm crown-rump length were studied.

The subclavian plexus is formed by fibres which come from that portion of the sympathetic trunk and its ganglia which are located in the root of

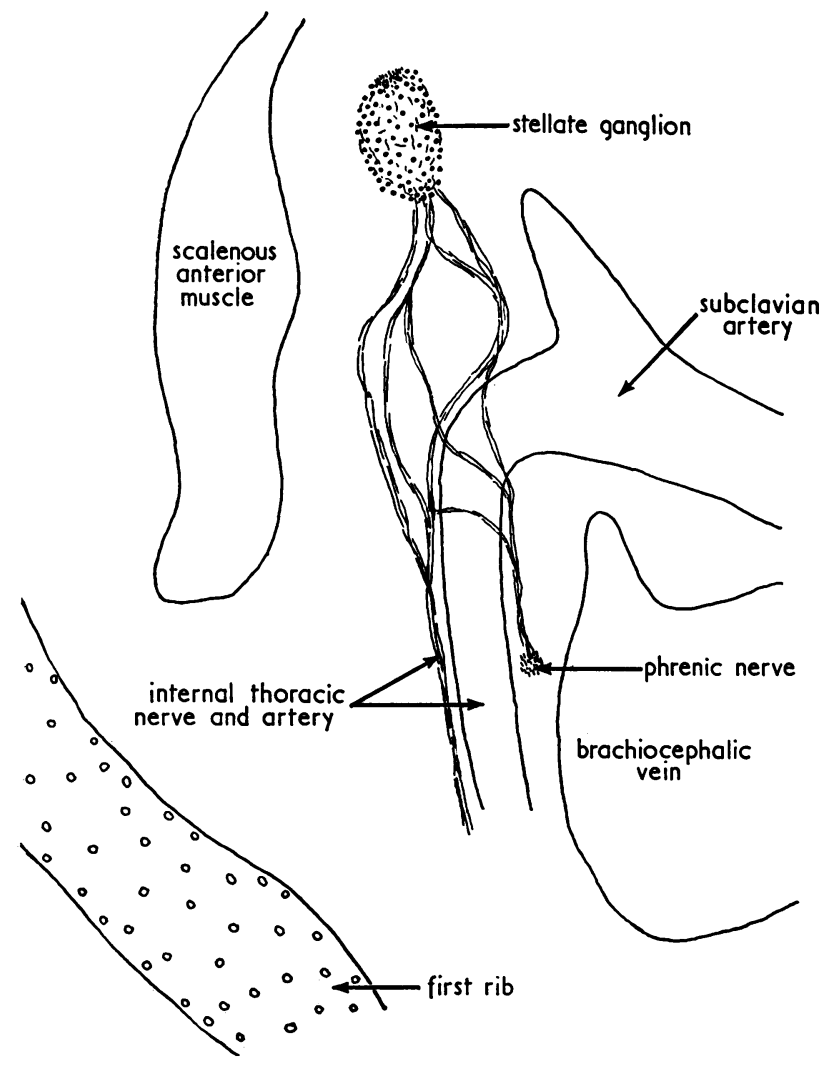

FIG. 1. A composite drawing from cross-sections of a $47 \mathrm{~mm}$ human fetus showing fibre bundles from the stellate ganglion passing to the internal thoracic nerve plexus and the phrenic nerve. Bodian method. $\times 51$ approximately. 


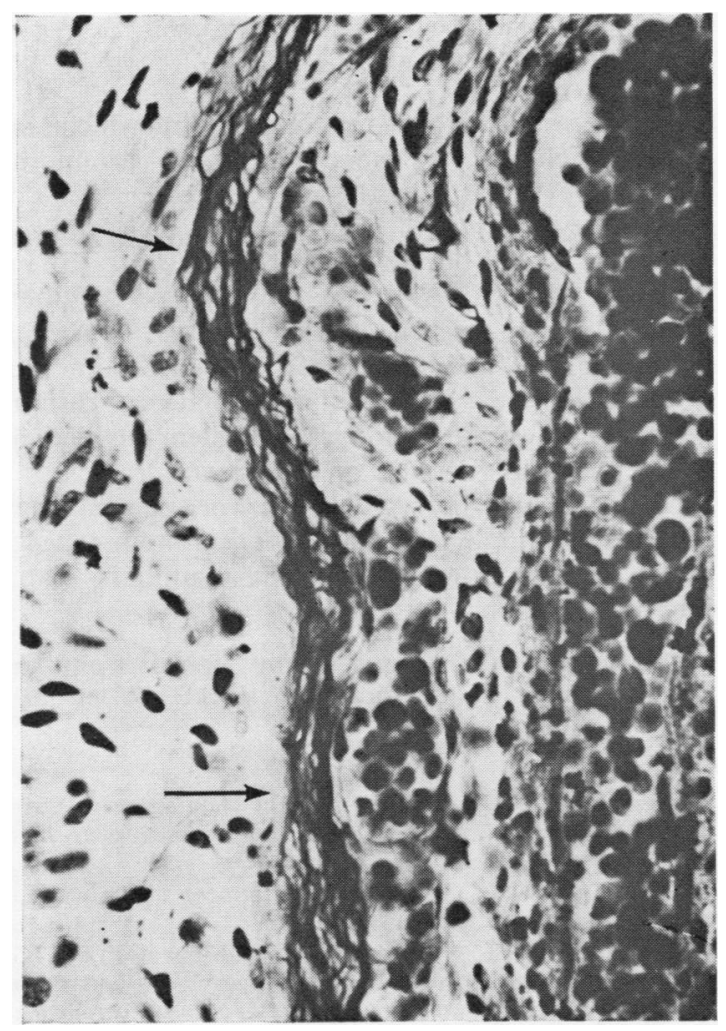

FIG. 2. A photomicrograph of a sagittal section of a $31 \mathrm{~mm}$ human fetus showing a nerve bundle in relation to the internal thoracic artery. Arrows point to a nerve bundle. Bodian method. $\times 250$.

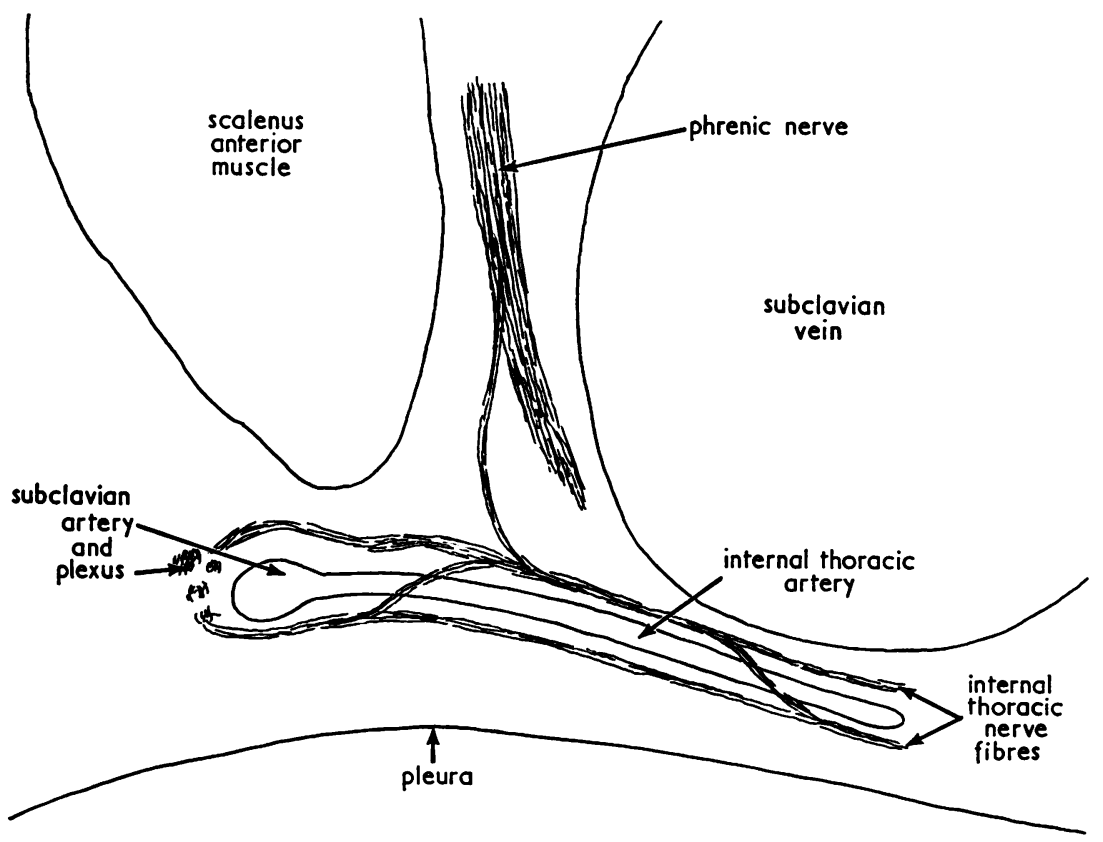

FIG. 3. A composite drawing from sagittal sections of a $31 \mathrm{~mm}$ human fetus showing the contribution of nerve fibres from the subclavian plexus and phrenic nerve to the internal thoracic nerve plexus. Bodian method. $\times 90$. 
the neck. This would include the inferior cervical sympathetic ganglion (or stellate ganglion) and the ansa subclavia (Kuntz, 1934). The subclavian plexus and the stellate ganglion contribute fibres to the nerve bundles (or nerve plexus) located along the internal thoracic (mammary) artery (Figs. 1 and 2). This plexus along the internal thoracic artery also receives fibres from the phrenic nerve (Fig. 3). These fibres can be traced along the internal thoracic artery to the level of the seventh costal cartilage where they are lost.

Some of the fibres of the internal thoracic nerve (or plexus) join the intercostal nerves and others follow closely along the perforating arteries. Some follow the anterior mediastinal arteries and are lost in the connective tissue in the region of the developing thymus. Those which follow the perforating arteries or join the intercostal nerves in the second, third, fourth, and fifth intercostal spaces distribute to the region of the anlage of the mammary gland.

The presence of nerve fibres along the internal thoracic artery may have clinical significance in the surgical treatment of myocardial ischaemia. In the Vineberg (1952) procedure the internal thoracic artery is dissected free between the first costal cartilage and the sixth interspace. This pedicle containing the internal thoracic artery is embedded in the wall of the heart. The implanted artery may form collateral connexions with branches of the coronary arteries in the revascularization of the heart. When this pedicle includes the vein and surrounding connective tissues, as in the Sewell procedure (Sewell, Sones, Fish, Joyner, and Effler, 1965), it would also include the internal thoracic nerve plexus. The implanted nerve fibres may play some role in the revascularization of the ischaemic heart.
The Sewell surgical procedure, in which the pedicle included the artery, vein, and surrounding connective tissues, resulted in a greater yield of functioning implants than those which utilized only the naked internal thoracic artery (Effler, Groves, Suarez, and Favaloro, 1967; Effler, 1969). In the absence of experimental data with regard to the function of the fibres in the internal thoracic nerve, we can only speculate concerning the effect these fibres may have when this pedicle is grafted into the wall of the heart.

The internal thoracic nerve (or plexus) may provide an important route for autonomic nerve fibres for the innervation of the mammary gland and the thymus.

This work was supported by grants from the Oregon Heart Association and U.S.P.H.S. 1 R01 NS08904-02.

\section{REFERENCES}

Bodian, D. (1936). A new method for staining nerve fibers and nerve endings in mounted paraffin sections. Anat. Rec., 65, 89.

Effler, D. B. (1969). The surgical treatment of myocardial ischemia. Clin. Symposia, 21, 3.

- Groves, L. K., Suarez, E. L., and Favaloro, R. G. (1967). Direct coronary artery surgery with endarterotomy and patch-graft construction. J. thorac. cardiovasc. Surg., 53, 93.

Kuntz, A. (1934). The Autonomic Nervous System, 2nd ed. Lea and Febiger, Philadelphia.

Pearson, A. A., and Whitlock, M. M. (1949). The use of a buffer in the silver gelatin stain for nerve fibers. Anat. Rec., 103, 581.

Sewell, W. H., Sones, F. M., Fish, R. G., Joyner, J. T., and Effler, D. B. (1965). The pedicle operation for coronary insufficiency: technique and preliminary results. $J$. thorac. cardiovasc. Surg., 49, 317.

Vineberg, A. (1952). Treatment of coronary artery insuffciency by implantation of the internal mammary artery into the left ventricular myocardium. J. thorac. Surg., 23, 42 . 